\title{
Best Quadratic unbiased Estimator for Variance Component of One-Way Repeated Measurement Model
}

\author{
Abdulhussein Saber AL-Mouel ${ }^{1}$ and Jasim Mohammed Ali Al-Isawi² \\ $1^{\text {st: }}$ Mathematics Department, College of Education for Pure Science AL-Basrah University-Iraq \\ abdulhusseinsaber@yahoo.com \\ $2^{\text {nd: }}$ Mathematics Department, College of Education for Pure Science AL-Basrah University-Iraq \\ Jas77im@gmail.com
}

\section{ABSTRACT}

The studies of analysis of variance components is one of the important topics in mathematical statistics for this subject of wide application. In this paper given best quadratic unbiased estimator of variance components for balanced data for linear one-way repeated measurement model (RMM). We computed the quadratic unbiased estimator, which has minimum variance (best quadratic unbiased estimate (BQUE)) by using analysis of variance (ANOVA) method of estimating the variance components.

Indexing terms/Keywords: Quadratic Estimator, Repeated Measurement Model, ANOVA Method, Variance Components, Quadratic Form, Estimation.

Language: English

Date of Submission: 2018-04-13

Date of Acceptance: 2018-04-20

Date of Publication: 2018-04-30

ISSN: 2347-1921

Volume: 14 Issue: 01

Journal: Journal of Advances in Mathematics

Website: https://cirworld.com

This work is licensed under a Creative Commons Attribution 4.0 International License.

\section{INTRODUCTION}

The variance component analogue of the best linear unbiased estimator of a function of effects is a best quadratic unbiased estimator (BQUE), that is, a quadratic function of the observations that is unbiased for the variance component and has minimum variance among all such estimators [8].

Repeated measurements is a term used to describe data in which the response variable for each experimental unit is observed on multiple occasions and possibly under different experimental conditions. There are variety of disciplines, which make use of repeated measurements as applications in the health and life sciences, epidemiology, biomedical research, education, psychology and the social sciences, etc. Repeated measurements 
occur frequently in observational studies, which are longitudinal in nature, and in experimental studies incorporating repeated measures designs. In the repeated measurements setting, independent factors or covariates may be classified into one of two categories: within-subject covariates and between-subject covariates. Within-subject covariates, also referred to as within-subject or within-unit factors, repeated measures factors and/or time-dependent covariates, represent variables or experimental conditions which vary over time within subjects or experimental units [9].

Several methods are available in literature for estimating the variance components. In this paper, we consider analysis of variance methods of estimating the variance components linear of the one-way repeated measurement model (RMM) to obtained BQUE for this model.

\section{SETTING THE MODEL}

Consider the linear model and parameterization for the one-way (RMM) with one between - units factor incorporating univariate random effects [1].

$$
Y_{i j k}=\mu+\tau_{j}+\delta_{i(j)}+\gamma_{k}+(\tau \gamma)_{j k}+\varepsilon_{i j k}
$$

where

$i=1, \ldots, n$ is an index for experimental unit within group $j$,

$j=1, \ldots, q$ is an index for levels of the between-units factor (group),

$k=1, \ldots, p$ is an index for levels of the within-units factor (time),

$Y_{i j k}$ is the response measurements at time $k$ for unit $i$ within group $j$,

$\mu$ is the overall mean,

$\tau_{j}$ is the added effect for treatment group $j$,

$\delta_{i(j)}$ is the random effect due to experimental unit $i$ within treatment group $j$,

$\gamma_{k}$ is the added effect for time $k$,

$(\tau \gamma)_{j k}$ is the added effect for the group $j \times$ time $k$ interaction, and

$\varepsilon_{i j k}$ is the random error on time $k$ for unit $i$ within group $j$.

For the parameterization to be of full rank, we impose the following conditions:

$$
\left.\begin{array}{l}
\sum_{j=1}^{q} \tau_{j}=0, \quad \sum_{k=1}^{p} \gamma_{k}=0, \\
\sum_{j=1}^{q}(\tau \gamma)_{j k}=0 \quad \forall k=1, \ldots, p, \quad \sum_{k=1}^{p}(\tau \gamma)_{j k}=0 \forall j=1, \ldots, q,
\end{array}\right\}
$$

We assume that the $\varepsilon_{i j k}$ 's and the $\delta_{i(j)}$ 's are independent with 


$$
\begin{gathered}
\varepsilon_{i j k} \sim \text { i.i.d } N\left(0, \sigma_{\varepsilon}^{2}\right) \text { and } \delta_{i(j)} \sim \text { i.i.d } N\left(0, \sigma_{\delta}^{2}\right) \\
\text { Let } \quad X=\operatorname{vec}(Y)=\left[\begin{array}{c}
Y_{111} \\
\vdots \\
Y_{n q p}
\end{array}\right]=\left[Y_{111}, \ldots, Y_{11 p}, Y_{1 q 1}, \ldots, Y_{n q 1}, \ldots, Y_{n q p}\right]^{\prime}
\end{gathered}
$$

Definition 1: The quadratic estimator $(Q E)$ of $\sigma_{\delta}^{2}$ is quadratic form which satisfies that:

$$
\hat{\sigma}_{\delta}^{2}=Q(A)=Y^{\prime} A Y
$$

For simply we denoted to $Q(A)$ by $\boldsymbol{A}$.

Let $\mathrm{A}=\left\{A: A\right.$ is symmetric square matrix such that $\left.Q(A)=\hat{\sigma}_{\delta}^{2}\right\}$

And $\mathbf{Q}$ is a set of any quadratic estimator (QE) of $\sigma_{\delta}^{2}$. i.e.

$$
\mathbf{Q}=\{\boldsymbol{A}=Q(A): \text { for any } A \in \mathrm{A}\} \text {. }
$$

Definition 2: The best quadratic unbiased estimate (BQUE) of $\sigma_{\delta}^{2}$ is a quadratic estimator $\boldsymbol{Q} \in \mathbf{Q}$ satisfies that : reshape

$$
\left.\begin{array}{l}
\text { (a) } E(\boldsymbol{Q})=\sigma_{\delta}^{2} \\
\text { (b) } \operatorname{var}(\boldsymbol{Q}) \leq \operatorname{var}\left(\boldsymbol{Q}^{*}\right) \text { for any } \boldsymbol{Q}^{*} \in \quad \boldsymbol{Q}
\end{array}\right\}
$$

Note: The general quadratic estimator for any $\boldsymbol{Q} \in \mathbf{Q}$ and $Q \in$ A be denoted by

$$
\boldsymbol{Q}=X^{\prime} Q X=\sum_{i j k ; r s t} Y_{i j k} Y_{r s t} q_{i j k}^{r s t} \quad \text { on reshape } Q=\left(q_{i j k}^{r s t}\right)
$$

3. Method of Analysis of variance (ANOVA)

Sum of square to groups, subjects (group), time, group $\times$ time (interaction) and residuals are given by:

$$
\begin{aligned}
& S S \tau=n p \sum_{j=1}^{q}\left(\bar{y}_{. j .}-\bar{y}_{. . .}\right)^{2}=X^{\prime}\left(\frac{J_{n}}{n} \otimes I_{q} \otimes \frac{J_{p}}{p}-\frac{J_{n}}{n} \otimes \frac{J_{q}}{q} \otimes \frac{J_{p}}{p}\right) X \\
& \left.\begin{array}{rl}
S S \delta & =p \sum_{i=1}^{n} \sum_{j=1}^{q}\left(\bar{y}_{i j .}-\bar{y}_{. j .}\right)^{2}=X^{\prime}\left(I_{n q} \otimes \frac{J_{p}}{p}-\frac{J_{n}}{n} \otimes I_{q} \otimes \frac{J_{p}}{p}\right) X \\
S S \gamma & =n q \sum_{j=1}^{q}\left(\bar{y}_{. . k}-\bar{y}_{. . .}\right)^{2}=X^{\prime}\left(\frac{J_{n}}{n} \otimes \frac{J_{q}}{q} \otimes I_{p}-\frac{J_{n}}{n} \otimes \frac{J_{q}}{q} \otimes \frac{J_{p}}{p}\right) X \\
S S \tau \gamma & =n \sum_{j=1}^{q} \sum_{k=1}^{p}\left(\bar{y}_{. j k}-\bar{y}_{. j .}-\bar{y}_{. . k}+\bar{y}_{. .)^{2}}\right. \\
& =X^{\prime}\left(\frac{J_{n}}{n} \otimes I_{q p}-\frac{J_{n}}{n} \otimes I_{q} \otimes \frac{J_{p}}{p}-\frac{J_{n}}{n} \otimes \frac{J_{q}}{q} \otimes I_{p}+\frac{J_{n}}{n} \otimes \frac{J_{q}}{q} \otimes \frac{J_{p}}{p}\right) X
\end{array}\right\}
\end{aligned}
$$




$$
\begin{aligned}
S S \varepsilon & =\sum_{i=1}^{n} \sum_{j=1}^{q} \sum_{k=1}^{p}\left(Y_{i j k}-\bar{y}_{. j k}-\bar{y}_{i j .}+\bar{y}_{. j .}\right)^{2} \\
& =X^{\prime}\left(I_{n q p}-\frac{J_{n}}{n} \otimes I_{q p}-I_{n q} \otimes \frac{J_{p}}{p}+\frac{J_{n}}{n} \otimes I_{q} \otimes \frac{J_{p}}{p}\right) X
\end{aligned}
$$

Where

$I_{p}$ denotes to the $p \times p$ identity matrix, $J_{p}$ denotes to $p \times p$ matrix of 1 's and $\otimes$ is kroncker prodect $\left\{\right.$ If $A=\left(a_{i j}\right)$ and $B=\left(b_{r s}\right)$ then $\left.A \otimes B=\left(A b_{r s}\right)\right\}$. And

$$
\begin{gathered}
\bar{y}_{. . .}=\frac{\sum_{i=1}^{n} \sum_{j=1}^{q} \sum_{k=1}^{p} Y_{i j k}}{n q p} \text { is the overall mean, } \\
\bar{y}_{. j .}=\frac{\sum_{i=1}^{n} \sum_{k=1}^{p} Y_{i j k}}{n p} \quad \text { is the mean for group } j, \\
\bar{y}_{i j .}=\frac{\sum_{k=1}^{p} Y_{i j k}}{p} \quad \text { is the mean for the } i^{t h} \text { subject in group } j, \\
\bar{y}_{. . k}=\frac{\sum_{i=1}^{n} \sum_{j=1}^{q} Y_{i j k}}{n q} \quad \text { is the mean for time } k, \\
\bar{y}_{. j k}=\frac{\sum_{i=1}^{n} Y_{i j k}}{n} \quad \text { is the mean for group } j \text { at time } k .
\end{gathered}
$$

The above results of equation ( 6 --10) can be summarized as ANOVA Table

Table 3.1 ANOVA for one-way Repeated measures model

\begin{tabular}{|c|c|c|c|c|}
\hline S.O.V. & Df & $S S$ & MS & EMS \\
\hline$\tau_{j}$ & $q-1$ & $S S \tau$ & $M S \tau=\frac{S S \tau}{q-1}$ & $\frac{n p}{q-1} \sum_{j=1}^{q} \tau_{j}^{2}+p \sigma_{\delta}^{2}+\sigma_{\varepsilon}^{2}$ \\
\hline$\delta_{i(j)}$ & $q(n-1)$ & $S S \delta$ & $M S \delta=\frac{S S \delta}{q(n-1)}$ & $p \sigma_{\delta}^{2}+\sigma_{\varepsilon}^{2}$ \\
\hline$\gamma_{k}$ & $p-1$ & $S S \gamma$ & $M S \gamma=\frac{S S \gamma}{p-1}$ & $\frac{n q}{p-1} \sum_{k=1}^{p} \gamma_{k}^{2}+\sigma_{\varepsilon}^{2}$ \\
\hline
\end{tabular}




\begin{tabular}{|c|c|c|c|c|}
\hline$(\tau \gamma)_{j k}$ & $(q-1)(p-1)$ & $S S \tau \gamma$ & $M S \tau \gamma=\frac{S S \tau \gamma}{(q-1)(p-1)}$ & $\frac{n}{(p-1)(q-1)} \sum_{\substack{\sigma_{\varepsilon}^{2} \\
+\sigma_{\varepsilon}}}^{q} \sum_{k=1}^{p}(\tau \gamma)_{j k}^{2}$ \\
\hline$\varepsilon_{\mathrm{ijk}}$ & $q(n-1)(p-1)$ & $S S \varepsilon$ & $M S \varepsilon=\frac{S S \varepsilon}{q(n-1)(p-1)}$ & $\sigma_{\varepsilon}^{2}$ \\
\hline Total & $n q p-1$ & $S S T$ & & \\
\hline
\end{tabular}

The variance components can be found by solving following equations

$$
\begin{aligned}
& M S \delta=p \sigma_{\delta}^{2}+\sigma_{\varepsilon}^{2} \\
& M S \varepsilon=\sigma_{\varepsilon}^{2}
\end{aligned}
$$

Moreover, the quadratic unbiased estimate of variance components are:

$$
\begin{gathered}
\hat{\sigma}_{\varepsilon}^{2}=M S \varepsilon \quad \text { and } \\
\because \quad E(M S \delta-M S \varepsilon)=p \sigma_{\delta}^{2}+\sigma_{\varepsilon}^{2}-\sigma_{\varepsilon}^{2}=p \sigma_{\delta}^{2} \\
\therefore \quad \hat{\sigma}_{\delta}^{2}=\frac{1}{p} E(M S \delta-M S \varepsilon)
\end{gathered}
$$

Now let $Q(M)$ is quadratic form given by ANOVA method of estimating $\sigma_{\delta}^{2}$.

From equation (7 and 10), we can write $\boldsymbol{M}(=Q(M))$ as:

$$
\begin{gathered}
M=X^{\prime} M X \\
=X^{\prime}\left(\frac{I_{n q} \otimes \frac{J_{p}}{p}-\frac{J_{n}}{n} \otimes I_{q} \otimes \frac{J_{p}}{p}}{q p(n-1)}-\frac{I_{n q p}-\frac{J_{n}}{n} \otimes I_{q p}-I_{n q} \otimes \frac{J_{p}}{p}+\frac{J_{n}}{n} \otimes I_{q} \otimes \frac{J_{p}}{p}}{q p(n-1)(p-1)}\right) X
\end{gathered}
$$

Then the matrix $M$ has this form

$$
\begin{aligned}
& M=\frac{I_{n q} \otimes \frac{J_{p}}{p}-\frac{J_{n}}{n} \otimes I_{q} \otimes \frac{J_{p}}{p}}{q p(n-1)}-\frac{I_{n q p}-\frac{J_{n}}{n} \otimes I_{q p}-I_{n q} \otimes \frac{J_{p}}{p}+\frac{J_{n}}{n} \otimes I_{q} \otimes \frac{J_{p}}{p}}{q p(n-1)(p-1)} \\
& M=\frac{1}{\eta}\left[I_{n q} \otimes J_{p}-\frac{J_{n}}{n} \otimes I_{q} \otimes J_{p}-I_{n q} \otimes \frac{J_{p}}{p}+\frac{J_{n}}{n} \otimes I_{q} \otimes \frac{J_{p}}{p}-I_{n q p}+\frac{J_{n}}{n} \otimes I_{q p}\right.
\end{aligned}
$$




$$
\begin{aligned}
& \left.+I_{n q} \otimes \frac{J_{p}}{p}-\frac{J_{n}}{n} \otimes I_{q} \otimes \frac{J_{p}}{p}\right] \\
\Rightarrow \quad M & =\frac{1}{\eta}\left[I_{n q} \otimes J_{p}-\frac{J_{n}}{n} \otimes I_{q} \otimes J_{p}-I_{n q p}+\frac{J_{n}}{n} \otimes I_{q p}\right] \\
= & \frac{1}{\eta}\left[\left(I_{n}-\frac{J_{n}}{n}\right) \otimes I_{q} \otimes\left(J_{p}-I_{p}\right)\right]
\end{aligned}
$$

Such that $\eta=q p(n-1)(p-1)$.

If a matrix $C$ have all elements of $M$ in (13) with arrangement as.

$$
C=(A+B) \otimes I_{q}
$$

$$
\text { And } \quad \boldsymbol{C}=Q(C)=X^{\prime} C X
$$

where $A=\frac{(n-1)}{n \eta}\left(J_{p} \otimes I_{n}-I_{n p}\right) \quad$ and $B=\frac{1}{n \eta}\left(J_{p}-I_{p}\right) \otimes\left(I_{n}-J_{n}\right)$

then $\boldsymbol{C}$ is an another quadratic unbiased estimator of $\sigma_{\delta}^{2},\left(E(\boldsymbol{C})=\sigma_{\delta}^{2}\right)$.

In addition, $C$ is a square and symmetric matrix of size $n q p \times n q p$.

The following relations can be obtained from matrix $C$ in (14) on rewrite as $C=\left(c_{i j k}^{r s t}\right)$

(a) $\sum_{i j k} c_{i j k}^{r s t}=0$ for any $r, s, t$.

(b) $\sum_{r s t} c_{i j k}^{r s t}=0$ for any $i, j, k$.

(c) $\sum_{j k} c_{i j k}^{r s t}=0$ for any $r, s, t, i$.

(d) $\sum_{s t} c_{i j k}^{r s t}=0$ for any $i, j, k, r$.

(e) $\sum_{i j k ; t} c_{i j k}^{i j t}=1$.

Theorem1: The BQUE estimator of $\sigma_{\delta}^{2}$ for model in (1) is given by ANOVA method,

that is, by quadratic form $Q(C)$ state in (15).

Proof:

Let $\boldsymbol{Q} \in \mathbf{Q}$ is unbiased estimator for $\sigma_{\delta}^{2}$

such that $\boldsymbol{Q}=\boldsymbol{C}+\boldsymbol{D}$

where 


$$
\begin{gathered}
\boldsymbol{D}=X^{\prime} D X=\sum_{i j k ; r s t} Y_{i j k} Y_{r s t} d_{i j k}^{r s t} \quad \text { and } \quad q_{i j k}^{r s t}=c_{i j k}^{r s t}+d_{i j k}^{r s t} \\
Q=\left(q_{i j k}^{r s t}\right), D=\left(d_{i j k}^{r s t}\right) \text { and } C=\left(c_{i j k}^{r s t}\right)
\end{gathered}
$$

In problem (17), by give requirement to each $q_{i j k}^{r s t}$ (since $\boldsymbol{Q}$ satisfies (4) and $c_{i j k}^{r s t}$ are known) then the problem(17) reduces to giving exact values to each $d_{i j k}^{r s t}$.

When $\boldsymbol{Q}=\boldsymbol{C}+\boldsymbol{D} \rightarrow E(\boldsymbol{Q})=E(\boldsymbol{C}+\boldsymbol{D})=\sigma_{\delta}^{2}$ since $E(\boldsymbol{Q})=\sigma_{\delta}^{2}$

this reduces to

$$
\begin{aligned}
\sum_{R}(\mu & \left.+\tau_{j}+\gamma_{k}+(\tau \gamma)_{j k}\right)\left(\mu+\tau_{s}+\gamma_{t}+(\tau \gamma)_{s t}\right)\left(c_{i j k}^{r s t}+d_{i j k}^{r s t}\right) \\
& +\sum_{R(r=i, s=j)} \sigma_{\delta}^{2}\left(c_{i j k}^{r s t}+d_{i j k}^{r s t}\right)+\sum_{i j k} \sigma_{\varepsilon}^{2}\left(c_{i j k}^{i j k}+d_{i j k}^{i j k}\right)=\sigma_{\delta}^{2}
\end{aligned}
$$

where index $R$ is refers to all indexes: $1 \leq(i, r) \leq n, 1 \leq(j, s) \leq q, 1 \leq(k, t) \leq p$.

And index $R(l)$ is refers to all indexes: $1 \leq(i, r) \leq n, 1 \leq(j, s) \leq q, 1 \leq(k, t) \leq p$.

Such that the restriction condition $l$ is satisfied.

From above equation (18) and by using (16 and 2) we have

$$
\begin{array}{ll}
\sum_{R} d_{i j k}^{r s t}=0 & \equiv \sum_{i j k ; r s t} d_{i j k}^{r s t}=0 \\
\sum_{R(r=i ; s=j)} d_{i j k}^{r s t}=0 & \equiv \sum_{i j k ; t} d_{i j k}^{i j t}=0 \\
\sum_{R(r=i ; s=j ; t=k)} d_{i j k}^{r s t}=0 \equiv \sum_{i j k} d_{i j k}^{i j k}=0
\end{array}
$$

Now since $E(\boldsymbol{Q})=\sigma_{\delta}^{2}$ then

$$
\operatorname{var}(\boldsymbol{Q})=E\left(\boldsymbol{Q}^{2}\right)-\sigma_{\delta}^{4}=E\left(\boldsymbol{C}^{2}\right)+E\left(\boldsymbol{D}^{2}\right)+2 E(\boldsymbol{C D})-\sigma_{\delta}^{4}
$$

We will study the term $(\boldsymbol{C D})$.

$$
E(\boldsymbol{C D})=E\left(\sum_{R} Y_{i j k} Y_{r s t} c_{i j k}^{r s t} \sum_{P} Y_{f g h} Y_{u v w} d_{f g h}^{u v w}\right)
$$

where index $P$ defined as $R$ but for subscripts $f, g, h, u, v, w$ it is refers to all indexes: $1 \leq(f, u) \leq$ $n, 1 \leq(g, v) \leq q, 1 \leq(h, w) \leq p$.

From (15) and (1)

$$
\because \boldsymbol{C}=\sum_{R} Y_{i j k} Y_{r s t} C_{i j k}^{r s t} \text { and } Y_{i j k}=\mu+\tau_{j}+\delta_{i(j)}+\gamma_{k}+(\tau \gamma)_{j k}+\varepsilon_{i j k}
$$


we have

$$
\begin{aligned}
& \boldsymbol{C}=\sum_{R}\left[\mu+\tau_{j}+\delta_{i(j)}+\gamma_{k}+(\tau \gamma)_{j k}+\varepsilon_{i j k}\right]\left[\mu+\tau_{s}+\delta_{r(s)}+\gamma_{t}+(\tau \gamma)_{s t}\right. \\
& \left.+\varepsilon_{r s t}\right] c_{i j k}^{r s t} \\
& \boldsymbol{C}=\sum_{R}\left[\delta_{i(j)}+\varepsilon_{i j k}\right]\left[\delta_{r(s)}+\varepsilon_{r s t}\right] c_{i j k}^{r s t}, \text { we using (16: c and d) } \\
& E(\boldsymbol{C})=E\left(\sum_{R}\left[\delta_{i(j)}+\varepsilon_{i j k}\right]\left[\delta_{r(s)}+\varepsilon_{r s t}\right] c_{i j k}^{r s t}\right) \\
& \therefore E(\boldsymbol{C D})=E\left(\sum _ { R } [ \delta _ { i ( j ) } + \varepsilon _ { i j k } ] [ \delta _ { r ( s ) } + \varepsilon _ { r s t } ] c _ { i j k } ^ { r s t } \quad \sum _ { P } \left[\mu+\tau_{g}+\delta_{f(g)}+\gamma_{h}\right.\right. \\
& \left.\left.+(\tau \gamma)_{g h}+\varepsilon_{f g h}\right]\left[\mu+\tau_{v}+\delta_{u(v)}+\gamma_{w}+(\tau \gamma)_{v w}+\varepsilon_{u v w}\right] d_{f g h}^{u v w}\right) \\
& =E\left(\sum _ { R } \sum _ { P } [ \delta _ { i ( j ) } + \varepsilon _ { i j k } ] [ \delta _ { r ( s ) } + \varepsilon _ { r s t } ] \left[\mu+\tau_{g}+\delta_{f(g)}+\gamma_{h}+(\tau \gamma)_{g h}\right.\right. \\
& \left.+\varepsilon_{f g h}\right]\left[\mu+\tau_{v}+\delta_{u(v)}+\gamma_{w}+(\tau \gamma)_{v w}\right. \\
& \left.\left.+\varepsilon_{u v w}\right] c_{i j k}^{r s t} d_{f g h}^{u v w}\right)
\end{aligned}
$$

From equations in [(2), (16) and (19)], since $\varepsilon_{i j k}$ 's and the $\delta_{i(j)}$ 's are independent with $\varepsilon_{i j k} \sim$ i.i.d $N\left(0, \sigma_{\varepsilon}^{2}\right)$ and $\delta_{i(j)} \sim$ i.i.d $N\left(0, \sigma_{\delta}^{2}\right)$ we have all terms of multiply the element of (22) equal to zero.

That is mean the equation in (20) becomes

when $E(\boldsymbol{C D})=0$ then we have

$$
\begin{aligned}
\operatorname{var}(\boldsymbol{Q}) & =E\left(\boldsymbol{Q}^{2}\right)-\sigma_{\delta}^{4}=E\left(\boldsymbol{C}^{2}\right)+E\left(\boldsymbol{D}^{2}\right)-\sigma_{\delta}^{4} \\
& =\operatorname{var}(\boldsymbol{C})+E\left(\sum_{i j k ; r s t}\left[Y_{i j k} Y_{r s t} d_{i j k}^{r s t}\right]^{2}\right)
\end{aligned}
$$

Then $\boldsymbol{Q}$ has minimum variance if $d_{i j k}^{r s t}=0$ for all $i, j, k ; r, s, t$. This clear implies to $\boldsymbol{Q}=\boldsymbol{C}$.

By above proof, we shown that, the BQUE of $\sigma_{\delta}^{2}$ is $\hat{\sigma}_{\delta}^{2}=\boldsymbol{C}=Y^{\prime} C Y$, where $C$ is given in equation (14) on rewrite as $C=\left(c_{i j k}^{r s t}\right)$.

And same manner we can found the $\mathbf{B Q} \boldsymbol{Q} \boldsymbol{E}$ for $\sigma_{\varepsilon}^{2}$, which equal to

$$
\left(\hat{\sigma}_{\varepsilon}^{2}=\boldsymbol{E}=Y^{\prime} E Y\right)
$$


where $E=\left(e_{i j k}^{r s t}\right)$, i.e $E$ is reshape of $\left(I_{n p}-\frac{J_{n}}{n} \otimes I_{p}-I_{n} \otimes \frac{J_{p}}{p}+\frac{J_{n p}}{n p}\right) \otimes I_{q}$.

\section{REFERENCES}

[1] AL-Mouel, A. H. S., A.J. Mohaisen and Khawla (2017). Bayesian One- Way Repeated Measurements Model Based on Bayes Quadratic Unbiased Estimator, Journal of Advance in Mathematics, Vol. 13, No. 2, pp. 71767182.

[2] AL-Mouel, A. H. S. and Wang, J. L. (2004). One-Way Multivariate Repeated Measurements Analysis of Variance Model. Applied Mathematics a Journal of Chinese Universities, 19, 4, 435-448.

[3] AL-Mouel, A. H. S. (2004). Nested repeated measures model sufficiency and estimation. Chinese Journal of Applied Probability and Statistics, 20, 133-146.

[4] Crump S. L. (1946), The estimation of variance components in the analysis of variance, Biometrics Bulletin ,Vol. 2 ,pp. 7-11.

[5] Graybill F.A. (1954), On quadratic estimates of variance components, Journal paper No. J-2-171 of the lowa Agricultural Experiment Station, Ames, lowa, pp. 367-372.

[6] James R. S. (2017), Matrix Analysis for Statistics ,Third Edition, John Wiley \& Sons, Inc., Canada.

[7] Rao, C. R. (1971b), Minimum variance quadratic unbiased estimation of variance components. J. Multivariate Anal., 1(4), 445-456.

[8] Sahai, H. and Ojeda, M. M. (2005), Analysis of variance for random models, Birkhäuser Boston, USA.

[9] Vonesh, E.F. and Chinchilli, V. M. (1997) linear and nonlinear models for the analysis of Repeated Measurements, Marcel Dakker, Inc. New York. 